\title{
Analyses and Review of the Impact of Social Networking
}

Bekim Fetaji
b.fetaji@seeu.edu.mk
Majlinda Fetaji
m.fetaji@seeu.edu.mk
Alajdin Abazi
* South East European University, Contemporary Sciences and Technologies, llindenska bb, 1200 Tetovo, Macedonia
$* *$ International Balkan University, Information Technology, Skopje, Macedonia
Mirlinda Ebibi
m.ebibi@ibu.edu.mk

Doi:10.5901/mjss.2015.v6n2s5p120

\section{Abstract}

Research study has focused on investigating: the ways in which people are engaging online using social networking to learn, inform and teach others, express themselves, challenge and create views on society and their place within it requires recognition that their online practices?, the benefits of SNS use are dependent on good internet and media literacy: having the skills to critically understand, analyse and create media content?, what are the range of risks and opportunities associated with the practices of social networking that must be understood and addressed by young people, policy makers and the community? The study focused primarily on investigating the benefits and falls-down of social networking services-SNS and trying to illuminate the ways in which the "online" and "offline" worlds are mutually constituted, potential opportunities to extend and improve. Maximizing the benefits of social networking and promoting internet and media literacy may help protect young people from many of the risks of online interaction, such as cyber-bullying, privacy breaches and predation. The research study is primarily trying to answer these research questions. Findings and recommendations are provided.

Keywords: Social networking, social media, impact of social media on the web, benefits of social networking

\section{Introduction}

The use of Social Networking Services (SNS) such as Facebook. com, Twitter. com, Google +plus, Linkedln and many others - has become a popular and integral part of everyday communication currently. Social networking according to (NMC's official Horizon Project, 2007) "is seen as a key opportunity to engage students more deeply into their course activities since these tools offer possibilities of contributing, sharing, communicating, and collaborating. "

Research in this area is an emerging field and studies identifying the negative impacts have tended to dominate the popular media and much policy development. However, there is substantial evidence of the benefits associated with SNS use, which has been largely neglected in public debate. The review report from (Beer, Dr David. , 2008) summarises current evidence concerning the enabling effects of SNS in the context of young people's everyday lives. Drawing on a range of sources this summary encompasses a variety of disciplines including education, sociology, political science, cultural studies and health. Policy currently focuses primarily on regulating the negative effects of SNS and social media, frequently framing digital citizenship within an online risk-management paradigm. This report finds that the benefits of social networking are largely associated with the participatory nature of the contemporary digital environment. Yet participation in creative content production, dissemination and consumption is largely overlooked in cyber safety frameworks. The emphasis on the risks of SNS use is exacerbated by limited intergenerational understanding of young people's ability to navigate online environments and narrow definitions of youth citizenship. Reconceptualising these 
challenges in terms of expanding young people's digital citizenship opens up the potential to maximise the wide range of substantive benefits associated with online communicative practices.

Strategies for maximizing the benefits of SNS use must be created and guided having in mind the evidences from the best practices. More targeted research needs to be undertaken to ensure specific emerging practices are properly understood so the positive effects of SNS can be leveraged. Given young people are often proficient users of online and networked technologies, this report finds that strategies which promote dialogue and position young people as SNS experts may help to open up new spaces for policy making, program development and, ultimately, safe and respectful online practices by young and old alike.

\section{Analyses and Review of Social Networking}

According to (Beer, Dr David. , 2008) Social networking services (SNS) include services such as Facebook. com, MySpace. com and Bebo. com which have many millions of members each. It also includes services, such as Elftown. com (for fans of fantasy and science fiction) and Ravelry. com (for fans of knitting!) with small numbers of members, often connected by a specific common interest. Furthermore, many services created for media sharing (e. g. Flickr for photo sharing, Last. FM for music listening habits and YouTube for video sharing) have incorporated profile and networking features and may be thought of as part of this wider conceptualisation of SNS themselves (Beer, Dr David. , 2008). Indeed, SNS in a Web 2. 0 environment have transformed processes of communication and social interaction particularly with the increasing integration of social media functionality to these services.

Social media is generally used to describe collaborative media creation and sharing on a fairly large scale (that can include SNS but also other participatory media activities such as news blogs) but can be extended to include smaller user-generated content networks or micro-communities (i. e. the 'small media' aspect of the current media environment), and things that sometimes fall outside SNS such as blogs/vlogs, podcasts, wikis, game modding1. The rapid uptake of both social media and SNS practices by young people signifies an important shift in young peoples" use of the net primarily for information and entertainment to one of communication. Young people are consuming, producing, sharing and remixing media. This has led to the claim that today's young people are "produsers" they actively produce and consume media (Bruns 2008). This participatory media environment enables young people to engage in creative content production, empowering them with new means of creating and sustaining connections with others. It has also opened up new debates on how to conceptualise and promote what has come to be termed cyber citizenship (Matthew $\mathrm{O}$. Jackson, 2008).

Drawing on articles and reports by academics, industry, non-government and government researchers, this summary encompasses a range of disciplines including education, sociology, political science, cultural studies and health. Whilst the report draws upon an international literature, the focus is on the Australian context.

Almost all young Australians are online with $90 \%$ of $16-29$ years olds using the internet daily (Nielsen 2010a: 139). They spend more time online (an average of 22 hours per week) than any other age group (Nielsen 2015). Many young people are able to access quality internet at home: $67 \%$ of households have internet access with $78 \%$ connected through broadband (ABS 2010). They are also early adopters in the mobile phone market with 97 per cent owning a mobile phone of which 64\% are internet capable (Nielsen 2010a: 50). Young people are increasingly using their mobile phones to access the internet (54\% of $16-29$ years olds), making it the fifth most common use of mobile phones in that age group (Nielsen 2010a: 51). In addition to downloading content, looking up information and emailing, mobile engagement with SNS is rapidly gaining popularity: $39 \%$ of SNS users access these services via their mobile devices (Nielsen 2010a: 63).

The importance of SNS in young people"s everyday lives is indisputable: $90 \%$ of 12 to 17 year olds, and $97 \%$ of 16 to 17 year olds, use SNS (ACMA 2009a: 8). SNS use is the number one online activity for 16 to 29 year olds with 83\% reporting they use them on a regular basis (Nielsen 2010a: 136). SNS allow users to communicate with others in many forms. The most frequently used forms of communication include: updating one"s own profile; commenting on photos or other posts; posting public messages to others or "wall" style messages; social network based instant messaging (Nielsen 2010a: 169). While the reading and writing of blogs remains in the top ten online activities carried out by young people, its popularity is decreasing particularly with the rise of micro-blogging practices, for example via Twitter and the Facebook"S "status" function, both of which are further enabled by the use of SNS via mobile phones (Sariola, J. 2003).

In addition to communication, SNS facilitate the creation and sharing of various forms of content including blogs, video, photos and more. In general, Australians are the world"s most prolific users of social media, and young people under 25 are the most active group when it comes to creating, updating and viewing social media (Nielsen 2010c).

In the following sections of this report, we outline a range of risks and opportunities associated with the practices of 
social networking that must be understood and addressed by young people, policy makers and the community. In doing so, we emphasise that SNS in and of themselves do not yield particular risks or benefits. Rather, it is the sets of communicative and creative practices in which SNS are embedded that lead to positive or negative impacts. We firstly outline the risks that must be managed, and then describe the diverse range of positive effects - at both the level of the individual and the community - arising from social networking practices. It is crucial to highlight that the risks and opportunities associated with social networking practices are interdependent. That is, promoting the positive impacts of social networking depends in large part on developing young people"s strategies and skills for managing online risks. Simultaneously though, as we discuss below, emerging research shows that fostering the positive social, cultural and educational impacts of social networking practices can work to effectively mitigate the potential risks.

The focus on risk and protecting children and young people from harm is often based on concerns that young people lack awareness of the public nature of the internet (Sariola, J. 2003). In addition to the threat of abuse, some fear that young people"s use of SNS can compromise the development and maintenance of supportive friendships and involvement in institutions traditionally understood as the embodiment of "communities", namely school, sports clubs, families etc (Boase, J. , Horrigan, J. B. , Wellman, B. \& Raine L. 2006). These concerns have dominated both public debate and policy-making in recent years.

There is some evidence that young people are aware of potential privacy threats online and many proactively take steps to minimise potential risks (Ball et al 2012). Research has indicated that online risks „are not radically different in nature or scope than the risks minors have long faced offline, and minors who are most at risk in the offline world continue to be most at risk online" (Sariola, J. 2003). Although the risks are real and the consequences can be extremely serious, experts emphasise that it is important not to overstate fears or understate the complexity of the challenge (Boase, J. , Horrigan, J. B. , Wellman, B. \& Raine L. 2006). Further, given that social networking practices are a routine part of many young people"s lives, we need to seek ways to promote the positive impacts of these. Limited intergenerational understanding of young people"s ability to navigate online environments can contribute to a disproportionate emphasis on the risks of SNS use (ACMA 2009c). Young people are often proficient users of online and networked technologies. Harnessing, expanding and promoting their skills and understandings of SNS may hold the key for overcoming the issues of concern.

The role of media literacy for digital literacy and cybersafety has been well established, although policy and practice has been slow to respond to new ways of thinking about media literacy in a digital world. Traditionally media literacy has been understood and taught in relation to mass media, addressing issues of media ownership, censorship and advertising. However, today"s online and networked media environment requires a more complex digital or web literacy that is often not explicitly taught in school. This environment requires that young people develop new skills to participate and stay safe in the new digital media environment. Consequently, there are a number of components to online media literacy (Jie Tang , Jimeng Sun, Chi Wang , Zi Yang, 2009), including:

- Technical literacy - for example, the knowledge and skills required to use a computer, web browser or particular software program or application;

- Critical content literacy - the ability to effectively use search engines and understand how they "order" information; who or what organisations created or sponsor the information; where the information comes from and its credibility and/or nature;

- Communicative and social networking literacy - an understanding of the many different spaces of communication on the web; the formal and informal rules that govern or guide what is appropriate behaviour; level of privacy (and therefore level of safe self-disclosure for each); and how to deal with unwanted or inappropriate communication through them;

- Creative content and visual literacy - in addition to the skills to create and upload image and video content this includes understanding how online visual content is edited and "constructed", what kind of content is appropriate and how copyright applies to their activities;

- Mobile media literacy - familiarity with the skills and forms of communication specific to mobile phones (eg: text messaging); mobile web literacy, and an understanding of mobile phone etiquettes.

Research indicates that the use of social networking services can support the development of media literacy. The creation and sharing of content on services such as MySpace has been seen to increase both young people"s "technical literacy", as they learn to use code to create their profiles, and "creative content and visual literacy" as they draw from and re-use media in appropriate ways for communication and self-expression (Sariola, J. 2003). Given written text, photos, animation, sounds, music, video clips are core components of SNS, young people develop a deeper understanding of the production, nature and use of various forms of content, which is otherwise missing from school curricula (Sariola, J. 2003). 
There is much interest from schools, TAFE and Universities in the potential of public SNS and social media such as blogs to leverage or complement formal educational activities and enhance learning outcomes (Hermann, H. \& Burns, J. M. 2010). Whilst e-learning frameworks are now integrated into most educational settings, the use of SNS is less comprehensively utilised. Access to SNS varies according to state and educational level with some states banning access to SNS and social media services altogether (Hermann, H. \& Burns, J. M. 2010). Consequently there is a dearth of evidence on the impact of SNS on young people"s formal education (Anderson 2007). Nevertheless, pilot projects and research are being rolled out which highlight both the potential and the need for social software, services and practices to be integrated into school and higher education frameworks (eg. Fitzgerald \& Steele 2008). SNS is also being used to extend opportunities for formal learning across geographical contexts. For example, within the Linking Latitudes program established by Tasmania"s Sacred Heart School and Pularumpi School on Melville Island, learners from both schools use instant messaging and Skype to share information about their cultures and work collaboratively. Using SNS, young people from the two schools interact with learners from over forty other schools (DEECD 2010). Additionally, SNS use between teachers and students can improve rapport and motivation and engagement with education (Mazer, Murphy \& Simonds 2007). Studies conducted in the workplace on the role of ICT in learning and development find:

- As a setting for sharing content \& creating/maintaining relationships, Web 2. 0 functionality facilitates peerbased \& self-directed learning;

- Young people in particular value social \& interactive opportunities for learning;

- Handheld technology is a particularly useful tool for workplace learning due to "regular accessibility" (D. J. Watts, P. S. Dodds, and M. E. Newman 2012);

- Access to virtual or online communities is more important than the physical education environment (education. au 2009);

- Online forums and SNS can support the continuation and extension of learning and discussion outside formal classroom setting (education. au 2009c);

- Peer based learning is a key characteristic of the way in which young people direct their own learning outside school \& formal organisations. This is characterised by a context of reciprocity, where participants feel they can both produce and evaluate knowledge \& culture (A. Gionis, E. Terzi, and P. Tsaparas. 2013); and,

- Young people expect interactivity "the Net Generation has been described as experiential, engaged, and constantly connected, with a strong need for immediacy" (A. Gionis, E. Terzi, and P. Tsaparas. 2013)

Evaluations of e-learning strategies have found SNS platforms allow for the extension of learning discussion outside the formal classroom setting, therefore promoting deeper learning as young people not only engage with the material for longer but are more likely to relate to it and incorporate it into their everyday lives (e. g. education. au 2009 \& Hermann, H. \& Burns, J. M. 2009). Finally, studies conducted on the use of hand held devices to deliver workplace learning demonstrated that "regular accessibility" means young people can access resources in a way that is both convenient and relevant to them. This in turn translates into increased levels of implementation into work practice (R. S. Burt 2001).

It is important to note that the educational benefits of SNS are not experienced equally by all young people. Certain groups of learners, such as Indigenous young people, those from low socio-economic backgrounds and those living in remote areas, face persistent challenges of internet access and literacy (ACMA 2009b). Maximising the benefits of SNS for these groups specifically requires addressing access and digital literacy. Yet, where access and skills are promoted, SNS and social media can enhance the interactions of marginalised young people with their teacher and increase their confidence in educational activities (Boyd, 2006)). Utilising different formats - for example, attaching a multi-media file or attaching clip art - enables individuals to articulate and explain themselves when faced with cultural, social, language or learning barriers (Z, Lovrekovic, T. , 2012).

Regarding Informal knowledge and skills SNS can facilitate learning and skill development outside formal learning environments by supporting peer-to-peer learning of knowledge and skills, collaboration, diverse cultural expression, the development of skills valued in the modern workplace, and a more empowered conception of citizenship (Ito, et. al. , 2006; Jenkins, 2007: 3). Furthermore, because of the high level of agency and personalisation involved, SNS can be particularly important learning spaces for young people who struggle in traditional educational settings (Fetaji et. al 2007). Beyond substantial educational benefits studies have shown that SNS support informal learning interests and needs such as online marketing, advanced IT and creative content production as well as parenting methods for young parents (Hermann, H. \& Burns, J. M. , 2009: 1220). Such studies show that SNS constitute new avenues for engaging young people in learning activities. When sharing content and creating/maintaining relationships young people engage in peerbased, self-directed and interactive learning (accessible from outside the classroom), essential for engagement and deep learning (Fetaji et al 2007). Furthermore, the knowledge and skills young people are learning through SNS are directly 
relevant to the "participatory web" in which "user generated content is now integral in a rapidly developing online business model that capitalises on the social networks, creativity and knowledge of its users", and this means that new business models are expected to emerge (see OECD 2007). This has led some to claim that the learning enabled via SNS and social media will have a direct bearing on their economic futures (Hermann, H. \& Burns, J. M. , 2009).

There remain important questions about the extent to which informal learning enabled by SNS impacts upon formal learning. Although it cannot be presumed that daily use of technology outside of formal educational contexts translates into meaningful use for learning (Fetaji et al 2007) SNS will provide the most benefit in terms of learning when there is integration of young people's SNS use in educational settings and their everyday lives.

Rapid uptake of digital technologies have opened up unprecedented possibilities for amateur users to create and distribute content (D. J. Watts, P. S. Dodds, and M. E. Newman. 2002) such that media "users" have become "produsers" (Bruns, 2008). User-generated content describes both the generation of "original" creative content and "remixed" content that creatively reworks or repurposes existing content. The interrelationship between SNS and social media has provided a key impetus (via platforms such as youtube. com and flickr. com) for the sharing of this self-generated content with broader networks. Young people in particular are more immersed in this participatory media environment than any other age-group. They now create and share their own 'small media' in their everyday communicative, creative and social activities.

Creative content sharing practices (such as blogs, animations, videos, photos and digital collages) form an increasingly integral part of young people"s communicative exchange and play a significant role in young people"s developing sense of identity and community. Creative content production and sharing empowers individual young people through the following demonstrated benefits:

- $\quad$ fostering the development of literacy and technical skills (D. J. Watts, P. S. Dodds, and M. E. Newman. 2002);

- developing a sense of aspiration, personal achievement and self-worth, and fostering further creativity and self-expression (Hermann, H. \& Burns, J. M. and Tacchi, 2005) - all of which are key predictors of wellbeing;

- encouraging exploration and experimentation with new or different aspects of their identity (Boyd \& Ellison, 2008); and,

- $\quad$ reinforcing aspects of identity, such as ethnicity or cultural background (Boyd \& Ellison, 2008).

The production and exchange of creative content also has demonstrated community-building effects. It can enhance the sense of community, belonging and connection that comes from a "shared history" of exchanging creative content (Boyd \& Ellison, 2008). Collaborative creative production, in turn, gives rise to a stronger sense of connection with others and the formation of strong communities. These communities may be enduring or more ephemeral in nature. However, evidence suggests that, either way, they have positive impacts on participants" sense of community and connection (Richardson, Third and MacColl, 2009).

Young people"s use of SNS is important for the strengthening and development of social relationships, particularly as the online and offline worlds converge.

Strengthening existing relationships Having positive interpersonal relationships is an important predictor of wellbeing (Boyd \& Ellison, 2008) and can buffer individuals from many of the key stressors that characterise the transition from childhood to adulthood (Boyd \& Ellison, 2008). Internet use, generally, has been found to strengthen young people"s existing interpersonal relationships (Boyd \& Ellison, 2008). Email, instant messaging and social networking can address new barriers young people may face to forming and maintaining positive social relationships. These barriers can include lack of safe, accessible and welcoming public places to gather, limited transport to get there, and time free of structured activities such as school and sport. SNS challenge these barriers because they are accessible 24/7, from different physical locations and via different technologies (eg. computer, mobile device) (boyd 2006). Furthermore, SNS play a critical role in overcoming the impact that high levels of mobility and complexity can have on long-term relationships. For example, studies have found that SNS helps young people who have recently transitioned from high-school to university to develop new relationships while maintaining their high-school friendships. In particular, those students with lower levels of satisfaction with university life and lower levels of self-esteem benefited the most from active use of Facebook (Boyd \& Ellison, 2008). Indeed, some forms of online social networking, such as instant messaging, usually involve much smaller groups of participants (often one-to-one communication) and are primarily used to maintain existing friendship networks (Boyd \& Ellison, 2008). SNS also play an important role in young people"s development and exploration of intimate relationships (boyd 2007). Once contact between young people in an intimate relationship outside of school hours usually occurred on the family phone which was shared and regulated by parents. Now SNS, along with the mobile phone, have provided a space in which this communication can occur (Boyd \& Ellison, 2008). 


\section{Analyses of the Impact of Social Networking}

Most research has focused on the role SNS play in the maintaining and strengthening of existing offline relationships. This research often suggests that those relationships which occur solely online, while important, are "weaker" (Boyd \& Ellison, 2008). However, for some young people, particularly those who are marginalised or otherwise socially isolated, online relationships provided a significant, and sometimes the only, opportunity for such socialisation. As a study of SNS for young people who suffer chronic illness and/or disability demonstrates, not only did it provide the opportunity to develop such friendships but participants described these friendships as "true friends" that were amongst their most dependable and enduring (Jie Tang, Jimeng Sun , Chi Wang , Zi Yang, 2009). This ability to connect with others with shared values, views, needs or experiences, can assist young people experiencing marginalisation to identify potential supportive connections in their local community. Another study demonstrated how Facebook helped young people with lower levels of social skills develop friendships online that then translated offline (V. Arnaboldi, A. Guazzini, and A. Passarella 2013).

SNS play a significant role in the formation of new forms of "collective identity". For example, studies have found that SNS can help young people who are sexually and gender diverse to meet people and learn from each other, creating the sense of belonging to a broader community (Jie Tang, Jimeng Sun , Chi Wang , Zi Yang, 2009). This sense of belonging and acceptance can mean that young people who may be more vulnerable to isolation - such as those with chronic illness or a disability - often remain members of an online community long after their initial impetus is gone (V. Arnaboldi, A. Guazzini, and A. Passarella 2013). .

Content sharing plays a major role in cultivating belonging and a sense of collective identity. Sharing written, visual or audio content on SNS that represents or portrays an individual or community experience invites others to engage and relate. Ito and Okabe (2005) use the term „ambient co-presence" to explain this phenomenon: an ongoing visual access to a small-scale communication cluster (or 'community') via spontaneous and everyday images uploaded to a collaborative media space. Such a mode of 'sharing' and 'connection' does not require text-based or real-time communication and can also mitigate feelings of social isolation (V. Arnaboldi, A. Guazzini, and A. Passarella 2013).

While research specifically on SNS use needs to be conducted, it has been argued that SNS functionality has a key role to play in strengthening social connectedness, self-efficacy, general knowledge and/or life skills and is critical for the promotion of young people"s overall mental health and wellbeing (Boyd \& Ellison, 2008). The personalisation of SNS profiles is not only important to young people"s relationship with others but is positively associated with the individual"s sense of self-efficacy or personal agency (Hermann, H. \& Burns, J. M. \& Tachhi, 2005). Young person"s sense of "ownership" over the online space also enhances their sense of empowerment and accomplishment which reinforces the sense of belonging and attachment to community (V. Arnaboldi, A. Guazzini, and A. Passarella 2013). There is a demonstrated positive relationship between young people"s use of social networking services and self-esteem (Boyd \& Ellison, 2008). It is also argued that a sense of community and belonging has the potential to promote young people"s resilience, giving them the ability to successfully adapt to change and stressful events (V. Arnaboldi, A. Guazzini, and A. Passarella 2013) This is an area that requires more large-scale research, particularly considering the over-emphasis within the public realm on the negative psychological effects of SNS and social media. However, overall, it appears that the social connections developed and fostered through SNS play an important role in promoting young people"s wellbeing (Boyd \& Ellison, 2008).

\section{Conclusions}

This study attempted to resolve some of the definitional and methodological difficulties encountered by previous researchers. It involved review and comparative analyses of Social Networking.

Contemporary learning theory suggests that individual learners differ in the way they learn and that learning must be tailored to the individual learner. Consequently, learning environments must have the flexibility to adapt themselves for the individual learner.

This review finds that there are a number of significant benefits associated with the use of SNS including: delivering educational outcomes; facilitating supportive relationships; identity formation; and, promoting a sense of belonging and self-esteem. Furthermore, the strong sense of community and belonging fostered by SNS has the potential to promote resilience, which helps young people to successfully adapt to change and stressful events. Importantly, the benefits of SNS use are dependent on good internet and media literacy: having the skills to critically understand, analyse and create media content. Maximising the benefits of SNS and promoting internet and media literacy may help protect young people from many of the risks of online interaction, such as cyber-bullying, privacy breaches and predation. For 
example, understanding how to produce creative content and manage the distribution of this content supports fully informed decision making and assessment of one's own, and others", privacy.

The debate over whether SNS only foster existing relationships or wether they are an important facilitator of new relationships is based on an assumed distinction between face-to-face and online communication. There is increasing evidence that young people experience "online" and "offline" social worlds as "mutually constituted" (Boyd \& Ellison, 2008) and flexibly combine a number of modes of techno-social interaction (Third and Richardson, 2010). Mobile and advanced technologies enable exchanging knowledge and skills that make learning collaborative and adaptive process of knowledge acquisition (Fetaji et al 2007). Another advantage of m-learning is the possibility of attending a video conference wireless from a classroom with no physical location and cable connections constraints.

Social Networking Services (SNS) are significant players in the Web 2. 0 environment, transforming communication practices, opening new spaces and processes of socialisation and impacting upon traditional social structures. These effects are particularly relevant for the most frequent users of SNS - young people. This new environment poses certain challenges and, like any setting for social interaction, has some inherent risks. However, this review suggests that these challenges and risks have been over-emphasised in recent years both in popular media and social research. Through increased internet and media literacy - ensuring all young people develop the skills to critically understand, analyse and create media content - these challenges can be overcome and risks mitigated in a way that ensures the many benefits of SNS can be realised. In other words, by maximising the benefits of SNS, whether it be their role in delivering educational outcomes, or facilitating supportive relationships, identity formation, or a sense of belonging and resiliency, many of the risks of online interaction, such as cyber-bullying, privacy breaches and predation, can be minimised. Strategies to this end must be underpinned by best practice evidence and more research should be undertaken to ensure that emerging practices and effects of SNS are understood and responded to.

This review of the benefits of SNS also points to some potential opportunities to extend and improve conceptualisations of "cybercitizenship" in policy and practice. Firstly, the concept of cybercitizenship, and its associated policies and programs, are almost exclusively focused on children and young people and therefore speak to what young people should "become" rather than what they already "are". It implies deficiency and fails to recognise that young people (and indeed children) are often more engaged, knowledgeable and proactive when it comes to safe and responsible online practices. Cybercitizenship, therefore, is a concept that would more usefully be applied to the community as a whole, rather than as a set of policies that target young people as requiring protecting - or protection from - in a digital landscape. Indeed, the Australian Communications and Media Authority has recognised that cybercitizenship policy should address a broader audience - acknowledging that adults would benefit from initiatives in online safety that have previously targeted children and young people (ACMA, 2009).

The second insight is that the positioning of cybercitizenship within an online risk-management paradigm (particularly within policy) is inherently limiting given the substantial range and substantive benefits associated with online practices. This paper finds that the benefits of social networking are largely associated with the participatory nature of the contemporary digital environment, yet participation in creative content production, dissemination and consumption is largely overlooked in cybercitizenship frameworks. Incorporating academic work on cybercitizenship and thus broadening the notion of cybercitizenship to encompass the ways in which people are engaging online to express themselves, challenge and create views on society and their place within it requires recognition that their online practices may challenge commonly held notions about childhood, youth, gender, ethnicity and so on. Importantly, these considerations should be informed by young people"s own experiences and perspectives. It may also present the need to incorporate new kinds of ethical and legal information relating to "citizen" rights and responsibilities.

Finally, the analyses of the benefits of SNS illuminates the ways in which the "online" and "offline" worlds are mutually constituted. However, cybercitizenship as a concept has a tendency to reinforce notions that there is a distinction between computer and web-mediated communication on the one hand and all other modes of communication on the other. If it is to retain relevance and deliver benefits to the community, our understanding of cybercitizenship must be more holistic, to fully encompass and resonate across all of the settings in which we live our lives - be that home, school, work, our local communities or our communities of interest.

\section{References}

A. Gionis, E. Terzi, and P. Tsaparas. Opinion maximization in social networks. arXiv preprint arXiv: 1301. 7455, 2013.

Bennett, L. W. (1998) „The uncivic culture: Communication, identity, and the rise of lifestyle politics", PS, Political Science and \& Politics, 31 (4): $740-761$.

Boase, J. , Horrigan, J. B. , Wellman, B. \& Raine L. (2006) The Strength of Internet Ties: The Internet and email aid users in maintaining 
their social networks and provide pathways to help when people face big decisions, Pew Internet \& American Life Project, Washington.

Boyd \& Ellison (2008) „Social Network Sites: Definition, History \& Scholarship“ in Journal of Computer- Mediated Communication 13: 210-230.

Boyd (2006) „Friends, Friendsters, \& MySpace Top 8: Writing community into being on social network sites" in First Monday 11 (12).

Bruns, A. (2008) Blogs, Wikipedia, Second Life, and Beyond: From Production to Produsage, Peter Lang, New York. Bukowski, W. M. , Newcomb, A. F. \& Hartup, W. W. (1996) The Company They Keep: friendship in childhood \& adolescence, Cambridge University Press, New York.

D. J. Watts, P. S. Dodds, and M. E. Newman. Identity and search in social networks. science, 296 (5571): 1302--1305, 2002.

Fetaji, B. \& Fetaji, M. (2007). Learning Management Systems Evaluation and Comparative Analyses. In G. Richards (Ed.), Proceedings of World Conference on E-Learning in Corporate, Government, Healthcare, and Higher Education 2007 (pp. 285-292). Chesapeake, VA: AACE.

Hermann, H. \& Burns, J. M. (2008) „Rethinking the digital divide: findings from a study of marginalised young people's information communication technology (ICT) use" in Youth Studies Australia 27 (4): 35-42.

Jie Tang , Jimeng Sun, Chi Wang , Zi Yang, Social influence analysis in large-scale networks, Proceedings of the 15th ACM SIGKDD international conference on Knowledge discovery and data mining, June 28-July 01, 2009, Paris, France

M. S. Granovetter. The Strength of Weak Ties. The American Journal of Sociology, 78 (6): 1360--1380, 1973.

Matthew O. Jackson, Social and Economic Networks, Princeton University Press, Princeton, NJ, 2008

N. E. Friedkin and E. C. Johnsen. Social influence and opinions. Journal of Mathematical Sociology, 15 (3--4): 193--206, 1990.

R. A. Hill and R. I. M. Dunbar. Social network size in humans. Human Nature, 14 (1): 53--72, 2003.

R. S. Burt. Structural Holes versus Network Closure as Social Capital. 2001.

Sariola, J. (2003). The Boundaries of University Teaching: Mobile Learning as a Strategic Choice for the Virtual University. In H. Kynaslahti \& P. Seppala (Eds. ), Mobile Learning (pp. 71-78). Helsinki: IT Press.

V. Arnaboldi, A. Guazzini, and A. Passarella. Egocentric Online Social Networks: Analysis of Key Features and Prediction of Tie Strength in Facebook. Computer Communications, 36 (10--11): 1130--1144, 2013.

Z, Lovrekovic, T. , (2012). "Software Application for Managing Multidisciplinary and Interdisciplinary Projects as a Part of Educational Process", Journal TEM, Technology Education Management Informatics, ISSN: 2217-8309, Volume 1, Number 1, 2012, Association for Information and Communication Technology Education and Sciences, Serbia. 